\title{
ASYMPTOTIC TRACTS OF HARMONIC FUNCTIONS I
}

\author{
K. F. BARTH and D. A. BRANNAN ${ }^{1,2}$
}

\section{Introduction}

This paper begins a study of the asymptotic tracts of functions that are either harmonic or subharmonic in the plane. An asymptotic tract of such a function $u(z)$ is a component of the set $\{z: u(z) \neq c\}$ for some $c \in R$; we will often, without loss of generality, suppose that $c=0$ and $u(z)>0$ in the tract. In this paper we deal with the relationship between the size ("angular width") of asymptotic tracts and the growth of the function itself; in a second paper we will emphasise the topological properties of tracts. Our main tools here will be a modification of a theorem of Tsuji [9; Theorem III. 67, p. 112], [10] to prove positive results and the AhlforsWarschawski distortion theorem to show that these results are best possible.

In Section 2 we prove:

Theorem 1. Let $u(z)$ be subharmonic, non-negative and non-constant in the plane. Suppose that every sufficiently large circle centred at the origin meets the set $\{z: u(z)=0\}$. Let $\left\{D_{k}\right\}$ be an enumeration of the family, $\mathscr{F}$, of components of $\{z: u(z)>0\}$. Then:

(a) Each component, $D_{k}$, is unbounded.

(b) $u$ is unbounded in each component $D_{k}$.

(c) Let the order, $\varrho_{k}$, of $u(z)$ in each tract $D_{k}$ be defined by the formula:

$$
\varrho_{k}=\limsup _{|z| \rightarrow \infty, z \in D_{k}} \frac{\log |u(z)|}{\log |z|} .
$$

Then $\sum_{k} 1 / \varrho_{k} \leqq 2$, where $1 / \varrho_{k}$ is taken as zero whenever $\varrho_{k}=+\infty$.

Theorem 1(c) can be thought of as an analogue of the Denjoy conjecture for harmonic functions (see remarks at end of Section 2), and is closely related to theorems of Kjellberg [5; Theorem II, p. 349] and Heins [3; Theorem 5.1, p. 74]. Sections 3 and 4 contain an array of examples showing that Theorem 1 is best possible; in particular we will prove:

1 The authors gratefully acknowledge support from the National Science Foundation.

2 Presented to the American Mathematical Society on January 6, 1978 and January 9, 1981. 
Theorem 2. Let $\varrho_{k}$ be defined as in Theorem 1(iii). Corresponding to each of the following situations there exists such a function harmonic in the entire plane:

(a) $\varrho_{1}=2$;

(b) $\sum_{k=1}^{n} 1 / \varrho_{k}=2$ for any given $n \geqq 2$;

(c) $\sum_{k=1}^{\infty} 1 / \varrho_{k}=2$, where infinitely many of the $\varrho_{k}$ are finite;

(d) For any given $\sigma$ with $0<\sigma<2$ and any given $n \geqq 1, \sum_{k=1}^{n} 1 / \varrho_{k}=\sigma$, where all other $\varrho$ 's are $+\infty$;

(e) For any given $\sigma$ with $0<\sigma<2, \sum_{k=1}^{\infty} 1 / \varrho_{k}=\sigma$ where the sum is taken over all finite $\varrho_{k}$ (of which there is an infinite number) and all other @'s are $+\infty$.

Some of the above examples can be constructed by other methods, but we prefer to use the Ahlfors-Warschawski approach since it provides a unified treatment of them all.

The next theorem is an extension of [1; Theorem 1, p. 363]. We make an assumption on the "angular width" of the tract, and thus obtain a more precise estimate of the growth of $u(z)$ on $\Gamma$ (see below). Note that (c) (below) is always satisfied if $\alpha=2$, so that our result includes [1; Theorem 1].

Theorem 3. Let the function $u(z)$ be subharmonic in an unbounded domain $D$ whose complement meets every sufficiently large circle centred at the origin, and let $\sigma \theta(\sigma)$ denote the length of the set $D \cap\{z:|z|=\sigma\}$. Suppose, in addition, that:

(a) $\lim _{z \rightarrow \xi}, \sup _{z \in D} u(z) \leqq 0$, where $\xi$ is any finite boundary point of $D$;

(b) $u\left(z_{0}\right)>0$ for some $z_{0} \in D$; and

(c) for some $r_{1} \geqq 0$, some $\alpha>0$ and all sufficiently large $r$

$$
\frac{1}{\log r} \int_{r_{1}}^{r} \frac{d \sigma}{\sigma \theta(\sigma)} \geqq \frac{1}{\alpha \pi} .
$$

Let $\varphi(r)$ be a positive increasing function of $r$ such that $\int^{\infty} \varphi(t) t^{-(1+1 / \alpha)} d t<+\infty$. Then there exists a path $\Gamma$ going to infinity in $D$ such that

$$
\liminf _{z \rightarrow \infty, z \in \Gamma} \frac{u(z)}{\varphi(|z|)}>0
$$

Just as in $[1 ;$ p. 368] we observe that we could choose $\Gamma$ in Theorem 3 such that

$$
\lim _{z \rightarrow \infty, z \in \Gamma} \frac{u(z)}{\varphi(|z|)}=+\infty .
$$

The theorem of Tsuji plays the same role in the proof of Theorem 3 that the Carleman-Milloux inequality played in the proof of [1; Theorem 1, p. 363]; it takes into account "how big" (in the angular sense) $C-D$ is.

Finally, in Section 6 we construct an example (Theorem 4) that shows that Theorem 3 is essentially best possible. 


\section{Proof of Theorem 1}

(a) Let $D$ be any member of $\mathscr{F}$. Then the function

$$
v(z)=\left\{\begin{array}{lll}
u(z) & \text { if } & z \in D \\
0 & \text { if } & z \notin D
\end{array}\right.
$$

is also subharmonic in the plane.

Assume, on the contrary, that $D$ is bounded, and choose an $R>0$ such that $D$ lies in $D_{R}=\{z:|z|<R\}$. Then $u(z)=0$ on $\partial D_{R}$, and so $u(z) \leqq 0$ in $D_{R}$ by the harmonic comparison principle. Hence $D=\emptyset$. This contradiction shows that $D$ must be unbounded.

(b) The Phragmén-Lindelöf generalised maximum principle [6], [8; p. 176] shows that since $u$ is non-constant in each $D_{k}$, then necessarily $u$ is unbounded above in each $D_{k}$.

It follows from a result of Talpur [7] that each $D_{k}$ must contain a path going to $\infty$ on which $u(z) \rightarrow+\infty$.

(c) Our main tool here is the following lemma, which is a straightforward modification of a result of Tsuji [9; Theorem III. 68, p. 117]:

Lemma 1. Let $D$ be a domain in the plane whose boundary meets every circle $\{z:|z|=r\}$ for each $r \geqq r_{0}$. Let $u(z)$ be a non-constant function subharmonic in $D$ such that $\lim _{\sup _{z} \in D, z \rightarrow \partial D} u(z) \leqq 0$, and let $x$ be any constant with $0<x<1$. Then if $u(z)$ is unbounded above in $D$,

$$
\log M_{D}(r) \geqq \pi \int_{r_{0}}^{\chi r} \frac{d t}{t \theta(t)}-c(x),
$$

where $M_{D}(r)=\sup \{u(z):|z|=r, z \in D\}, t \theta(t)$ is the length of the set $D \cap\{z:|z|=t\}$, and $c(\varkappa)$ is some constant depending only on $\varkappa$.

First choose any positive integer $n$; then choose some $r_{0}=r_{0}(n)>0$ such that the components $D_{k}(1 \leqq k \leqq n)$ in $\mathscr{F}$ all meet $\left\{z:|z|=r_{0}\right\}$. Since $u(z)$ is unbounded in $D_{k}$, it follows from Lemma 1 (with $\varkappa=1 / 2$ ) that

$$
\log M_{D_{k}}(r) \geqq \pi \int_{r_{0}}^{r / 2} \frac{d t}{t \theta_{k}(t)}-c_{n},
$$

where the constant $c_{n}$ depends on the choice of $r_{0}$, and so on $\min \left\{|z|: z \in \partial D_{k}\right.$, $1 \leqq k \leqq n\}$ - that is, on $n$.

Take any $\varepsilon>0$. It follows from the definition of $\varrho_{k}$ that for all sufficiently large $r$

so that, for $r>2 r_{0}$,

$$
\log M_{D_{k}}(r)<\left(\varrho_{k}+\varepsilon\right) \log r,
$$

$$
\left(\varrho_{k}+\varepsilon\right) \log r>\pi \int_{r_{0}}^{r / 2} \frac{d t}{t \theta_{k}(t)}-c_{n} .
$$


Now since $0<\theta_{k}(t) \leqq 2 \pi$ for $t>r_{0}$, it follows that

$$
\pi \int_{r_{0}}^{r / 2} \frac{d t}{t \theta_{k}(t)} \geqq \pi \int_{r_{0}}^{r / 2} \frac{d t}{2 \pi t}=\frac{1}{2} \log \left(\frac{r}{2 r_{0}}\right) \rightarrow \infty \quad \text { as } \quad r \rightarrow \infty .
$$

Hence as $r$ becomes large we have that

$$
\left(\varrho_{k}+\varepsilon\right) \log r>\pi \int_{r_{0}}^{r / 2} \frac{d t}{t \theta_{k}(t)}\left[1+O\left(\frac{1}{\log r}\right)\right],
$$

which we can rewrite as

$$
\frac{1}{\varrho_{k}+\varepsilon}<\frac{\log r}{\pi}\left[1+O\left(\frac{1}{\log r}\right)\right] / \int_{r_{0}}^{r / 2} \frac{d t}{t \theta_{k}(t)} .
$$

Now' by the Cauchy-Schwarz inequality

so that

$$
\left(\int_{r_{0}}^{r / 2} \frac{d t}{t}\right)^{2} \leqq\left(\int_{r_{0}}^{r / 2} \frac{\theta_{k}(t)}{t} d t\right)\left(\int_{r_{0}}^{r / 2} \frac{d t}{t \theta_{k}(t)}\right)
$$

$$
1 / \int_{r_{0}}^{r / 2} \frac{d t}{t \theta_{k}(t)} \leqq \frac{1}{\log ^{2}\left(r / 2 r_{0}\right)} \int_{r_{0}}^{r / 2} \frac{\theta_{k}(t)}{t} d t
$$

Substituting this upper bound into (1) and summing over $1 \leqq k \leqq n$ for any $n$, we deduce that

$$
\begin{aligned}
\sum_{k=1}^{n} \frac{1}{\varrho_{k}+\varepsilon} & <\frac{\log r \cdot[1+O(1 / \log r)]}{\pi \log ^{2}\left(r / 2 r_{0}\right)} \int_{r_{0}}^{r / 2}\left(\sum_{k=1}^{n} \theta_{k}(t)\right) \frac{d t}{t} \\
& =\frac{2 \log r \cdot[1+O(1 / \log r)]}{\log \left(r / 2 r_{0}\right)} .
\end{aligned}
$$

Letting $r \rightarrow \infty$ gives that $\sum_{k=1}^{n} 1 /\left(\varrho_{k}+\varepsilon\right) \leqq 2$; and, since $\varepsilon>0$ is arbitrary, that $\sum_{k=1}^{n} 1 / \varrho_{k} \leqq 2$. Since $n$ is arbitrary, the result follows.

Remark 1. The function $u(z)=\log ^{+}|z|$ shows that for the result of Theorem 1(c) to hold some hypothesis such as that about 'all sufficiently large circles' must be necessary.

Remark 2. Since for any function $U(z)$ harmonic in the whole plane the function $u(z)=|U(z)|$ is subharmonic, it follows that the result of Theorem 1(c) can be thought of as an analogue for harmonic functions of the Denjoy conjecture for entire functions.

Remark 3. The function $u(z)=\left|\operatorname{Re}\left(z^{n / 2}\right)\right|, n \in N$, is subharmonic in $C$; here $\mathscr{F}$ consists of $n$ components in each of which the order of $u$ is $n / 2$. This shows that equality can occur in (c). 


\section{Proof of Theorem 2(a)}

Firstly we will construct a harmonic function in an unbounded domain in the plane; then the function harmonic in the plane that is obtained by approximating the earlier function via Arakelian's theorem has the desired property.

3.1. The auxiliary mappings. Let $C_{1}$ be a curve in the $t$-plane whose polar equation (in terms of $(r, \psi)$-polar coordinates) is given by $t=r e^{ \pm i \psi(r)} \quad(r \geqq 0)$ where $\psi(r)=\pi-(1 / 2) \Theta(r)$ for some function $\Theta(r)$ continuous on $[0, \infty)$ with the following properties:

(i) $\Theta(0)=2 \pi, \quad \lim _{r \rightarrow \infty} \Theta(r)=0$;

(ii) $\Theta(r) \in C^{1}([0, \infty))$;

(iii) $\Theta(r)$ is strictly decreasing on $[0, \infty)$;

(iv) $r \Theta^{\prime}(r) \rightarrow 0$ as $r \rightarrow \infty$;

(v) $\int^{\infty} r \Theta^{\prime}(r)^{2} d r<+\infty$;

(vi) $\int^{\infty} r^{-1} \Theta(r) d r<+\infty$.

[For example, $\Theta(r)=2 \pi /(1+r)^{\varepsilon}$ is quite satisfactory for any choice of $\varepsilon>0$.]

For use in what follows we first examine one consequence of conditions (i), (iii) and (vi) on the function $\Theta$. Since from (i) and (iii),

$$
0<\frac{\Theta(\varrho)}{\varrho[1-(1 / 2 \pi) \Theta(\varrho)]}<\frac{\Theta(\varrho)}{\varrho[1-(1 / 2 \pi) \Theta(1)]}
$$

for $1<\varrho<\infty$, it follows from (vi) that

Now define

$$
\int^{\infty} \frac{\Theta(\varrho)}{\varrho[1-(1 / 2 \pi) \Theta(\varrho)]} d \varrho<+\infty .
$$

$$
I(r)=\int_{1}^{r} \frac{\Theta(\varrho)}{\varrho[1-(\mathbb{1} / 2 \pi) \Theta(\varrho)]} d \varrho .
$$

Since the above integrand is positive, $I(r)$ is strictly increasing; and as it is bounded above, there must exist some $\mu>0$ such that

$$
I(r) \rightarrow \mu \quad \text { as } \quad r \rightarrow \infty .
$$

Next, let $D_{1}$ be the component of $C-C_{1}$ that contains the point 1. We can map $D_{1}$ onto a 'strip-like' domain $D_{2}$ in the $z$-plane by means of the mapping

$$
z=\frac{1}{2} \log t=\frac{1}{2}\{\log |t|+i \operatorname{Arg} t\},
$$

where we take the principal values of $\log$ and Arg. Hence if $z=x+i y$ and $|t|=r$, we 
have that

$$
x=\frac{1}{2} \log r, \quad y=\frac{1}{2} \operatorname{Arg} t .
$$

Since $D_{1}$ is symmetric in the real axis, so is $D_{2}$; indeed:

$$
D_{2}=\left\{z=x+i y: 2|y|<\pi-(1 / 2) \Theta\left(e^{2 x}\right)\right\} .
$$

Notice that $0, \infty$ as boundary points of $D_{1}$ correspond to $-\infty, \infty$ as boundary points of $D_{2}$.

Next, let $w=w(z)$ map $D_{2}$ onto the domain

$$
D_{3}=\{w=u+i v:|v|<\pi / 2\}
$$

with $-\infty,+\infty$ corresponding in each case. Finally we map $D_{3}$ onto the domain

$$
D_{4}=\{\zeta=\xi+i \eta: \xi>-1\}
$$

with $-\infty$, $+\infty$ as boundary points of $D_{3}$ corresponding to $-1, \infty$ as boundary points of $D_{4}$ by means of the mapping

$$
\zeta=e^{w}-1
$$

First, we have to determine the pre-image, $\Gamma_{1}$, in $D_{1}$ of the line $\Gamma_{4}=\{\zeta: \operatorname{Re} \zeta=0\}$ in $D_{4}$. For the mapping $D_{2} \rightarrow D_{3}$, we see from the Ahlfors-Warschawski distortion theorem [11] - assuming that certain conditions are satisfied - that

$$
w(x+i y)=\lambda+o(1)+\pi \int_{0}^{x} \frac{d s}{\theta(s)}+\pi i \frac{y}{\theta(x)}
$$

(as $x \rightarrow \infty$, uniformly in $y$ ), where $\lambda$ is some real constant and $\theta(x)=\pi-(1 / 2) \Theta\left(e^{2 x}\right)$.

The two conditions that must be satisfied for (4) to hold are:

(i) $\theta^{\prime}(x) \rightarrow 0$ as $x \rightarrow+\infty$, and

(ii) $\int^{\infty} \frac{\theta^{\prime}(x)^{2}}{\theta(x)} d x<+\infty$.

In (i) ; $\theta^{\prime}(x)=-e^{2 x} \Theta^{\prime}\left(e^{2 x}\right)$; it follows from our hypothesis (iv) on the function $\Theta$ that (i)' is indeed satisfied.

In (ii)', we have that

$$
\int^{\infty} \frac{\theta^{\prime}(x)^{2}}{\theta(x)} d x=\int^{\infty} \frac{e^{4 x} \Theta^{\prime}\left(e^{2 x}\right)^{2}}{\pi-(1 / 2)} \overline{\Theta\left(e^{2 x}\right)} d x=\frac{1}{2} \int^{\infty} \frac{r \Theta^{\prime}(r)^{2}}{\pi-(1 / 2) \Theta(r)} d r
$$

it follows from (5) and our hypothesis (v) on $\Theta$ that requirement (ii)' is also satisfied. Consequently the formula (4) is valid.

Under the mappings between the various domains, let us specify that the following points correspond:

$$
\begin{cases}0+i \eta & \text { in } D_{4}, \quad u+i v \text { in } D_{3}, \\ x+i y & \text { in } D_{2} \text { and } r \exp [ \pm i(\pi-\varepsilon(r))] \text { in } D_{1} .\end{cases}
$$


We take this last expression to be our definition of $\varepsilon(r)>0$. Part of our work will be to show that, for large values of $|\eta|, \varepsilon(r)$ is very small; notice that, by symmetry, it is sufficient to consider only positive values of $\eta$.

Now for the mapping between the points in (6) in $D_{3}$ and $D_{4}$ we have that

$$
e^{u+i v}-1=0+i \eta
$$

so that $e^{u} \cos v=1$ and $e^{u} \sin v=\eta$. Hence on the pre-image in $D_{3}$ of $\Gamma_{4} \cap\{\zeta: \operatorname{Re} \zeta>0\}$ we have

$$
\cos v=e^{-u} \text { and } \sin v=\left(1-e^{-2 u}\right)^{1 / 2} .
$$

Next, for the mapping between the points in (6) in $D_{1}$ and $D_{2}$ we have that

$$
x=\frac{1}{2} \log r \quad \text { and } \quad y=\frac{1}{2}[\pi-\varepsilon(r)],
$$

where we have used the fact that the upper half of $\Gamma_{4}$ must correspond to a part of $\Gamma_{1}$ in the upper half-plane.

Finally we see from (4) that for the mapping between the points in (6) in $D_{2}$ and $D_{3}$ we have that

$$
\left\{\begin{array}{l}
u=\lambda+o(1)+\pi \int_{0}^{x} \frac{d s}{\pi-(1 / 2) \Theta\left(e^{2 s}\right)} \text { and } \\
v=o(1)+\pi \frac{y}{\pi-(1 / 2) \Theta\left(e^{2 x}\right)} .
\end{array}\right.
$$

From (8) and (9) we see that

so that

$$
v=o(1)+\frac{\pi}{2} \cdot \frac{\pi-\varepsilon(r)}{\pi-(1 / 2) \Theta(r)}=o(1)+\frac{\pi}{2}-\frac{\pi}{2} \cdot \frac{\varepsilon(r)-(1 / 2) \Theta(r)}{\pi-(1 / 2) \Theta(r)},
$$

$$
\cos v=\sin \left\{o(1)+\frac{\pi}{2} \cdot \frac{\varepsilon(r)-(1 / 2) \Theta(r)}{\pi-(1 / 2) \Theta(r)}\right\}=\sin \left\{o(1)+\frac{1}{2} \varepsilon(r)\right\} \quad \text { (for } r \text { large), }
$$

since $\Theta(r) \rightarrow 0$ as $r \rightarrow \infty$ (by (i)). Since $0<(1 / 2) \varepsilon(r)<(\pi / 2)$ it follows that

$$
\cos v=o(1)+\frac{1}{2} \varepsilon(r) \quad(r \rightarrow \infty) .
$$

Next, we see from the first equations in each of (7) and (9) that

$$
\begin{aligned}
e^{-u} & =\exp \{-\lambda+o(1)\} \exp \left\{-\int_{0}^{x} \frac{d s}{\pi-(1 / 2) \Theta\left(e^{2 s}\right)}\right\} \\
& =\exp \{-\lambda+o(1)\} \exp \left\{-\int_{1}^{r} \frac{d \varrho}{2 \varrho[1-(1 / 2 \pi) \Theta(\varrho)]}\right\},
\end{aligned}
$$


where we have used the substitution $\varrho=e^{2 s}$; consequently,

$$
\begin{gathered}
\frac{e^{-u}}{r^{-1 / 2}}=\exp \{-\lambda+o(1)\} \exp \left\{-\int_{1}^{r} \frac{(1 / 2 \pi) \Theta(\varrho)}{2 \varrho[1-(1 / 2 \pi) \Theta(\varrho)]} d \varrho\right\} \\
\rightarrow \exp \left(-\lambda-\frac{1}{4 \pi} \mu\right)=L, \text { say },
\end{gathered}
$$

where $0<L<1$. In other words,

$$
e^{-u} \sim L r^{-1 / 2} \quad(\text { as } r \rightarrow \infty) \text {. }
$$

Substituting from (10) and (12) into (7) we get that

$$
o(1)+\frac{1}{2} \varepsilon(r)=\cos v=e^{-u} \sim L r^{-1 / 2} \quad(r \rightarrow \infty),
$$

and so in particular that

$$
\varepsilon(r) \rightarrow 0 \quad(\text { as } r \rightarrow \infty) .
$$

Now let the composition function mapping $D_{1}$ onto $D_{4}$ be denoted by

$$
f_{1}(t)=\xi_{1}(t)+i \eta_{1}(t)
$$

Then by Arakelian's theorem [2] there exists an entire function $f(t)=\xi(t)+i \eta(t)$ with the property that

$$
\left|f(t)-f_{1}(t)\right| \leqq e^{-\varphi(|t|)} \quad\left(t \in \bar{D}_{1}\right)
$$

for any pre-assigned increasing function $\varphi$ with $\int^{\infty} \varphi(x) x^{-3 / 2} d x<+\infty$. Without loss of generality we may suppose that $\varphi(0)>\log _{e} 10$, so that it follows from (14) that

$$
\left|\xi(t)-\xi_{1}(t)\right|<\frac{1}{10} \quad\left(t \in \bar{D}_{1}\right) .
$$

In particular we can deduce that $\xi(t) \geqq-11 / 10$ everywhere in $\bar{D}_{1}$.

3.2. The approximation $\xi(t)$. Let the set $D_{1}^{\prime}$ be defined to be

$$
D_{1}^{\prime}=\left\{t: t \in D_{1}, \xi_{1}(t)>0\right\} .
$$

Then $\partial D_{1}^{\prime}$ is the pre-image under a one-one map of the Jordan curve $\{\zeta: \operatorname{Re} \zeta=0\}$, which is a connected point set; hence $\partial D_{1}^{\prime}$ is also a connected set and a single Jordan curve from $\infty$ to $\infty$. Hence $D_{1}^{\prime}$ is unbranched in the sense that given any point $t_{1}^{\prime}$ in $D_{1}^{\prime}$ all curves in $D_{1}^{\prime}$ from $t_{1}^{\prime}$ to $\infty$ are homotopic in $D_{1}^{\prime}$. [An equivalent definition of $D_{1}^{\prime}$ being unbranched is that its complement is an unbounded continuum.]

Further, we know that $\xi(t)$ must be unbounded in $D_{1}$ since $\xi_{1}(t)$ is unbounded in $D_{1}$. Thus there exist points in $D_{1}$ where $\xi_{1}(t)>1 / 10$ and so necessarily $\xi(t)>0$; it follows that $D^{\prime \prime}=\left\{t: t \in D_{1}, \xi(t)>0\right\}$ is non-empty. We now check, in turn, the validity of the following three assertions: 
(i)" $\bar{D}^{\prime \prime} \subset D_{1}$;

(ii)" $D^{\prime \prime}$ has only one component; and

(iii)" $D^{\prime \prime}$ is unbranched.

To begin with we know from the definition of $D^{\prime \prime}$ that $D^{\prime \prime} \subset D^{\prime}$. If in fact $\bar{D}^{\prime \prime} \propto D_{1}$, there would exist some point $t^{\prime \prime} \in \partial D^{\prime \prime} \cap \partial D_{1}$, and so $\xi\left(t^{\prime \prime}\right)=0$ and $\xi_{1}\left(t^{\prime \prime}\right)=-1$; consequently $\left|\xi\left(t^{\prime \prime}\right)-\xi_{1}\left(t^{\prime \prime}\right)\right|=1$, which is impossible in view of (15). This completes the proof of (i)".

Next, we can use an argument similar to that above in the case $c=0$ to show that each of the sets

$$
\left\{t: t \in D_{1}, \xi_{1}(t)>c\right\}, c= \pm 1 / 10,
$$

is an unbranched and unbounded Jordan domain. We now consider the open set

$$
S=\left\{t: t \in D_{1}, \xi_{1}(t)>1 / 10\right\}
$$

in view of (15), $\xi(t)>0$ in $S$; hence there exists a component of $D^{\prime \prime}$, call it $D_{(1)}$ say, that contains $S$. Let $D_{(2)}$ be any other component of $D^{\prime \prime}$; since $D_{(1)} \cap D_{(2)}=\varphi$, we must have

$$
D_{(2)} \subset\left\{t: t \in D_{1},-1 / 10<\xi_{1}(t)<1 / 10\right\} .
$$

On $\partial D_{(2)}$ we must have $\xi(t)=0$ and inside $D_{(2)}$ we have $\xi(t)>0$. But since $D_{(2)}$ is a component of $D^{\prime \prime}$ and $\xi(t)$ is harmonic in the whole plane, it follows (as in Theorem 1(a), (b)) that $D_{(2)}$ must be unbounded and that $\xi(t)$ must be unbounded above in $D_{(2)}$. Hence there must exist some point $t_{(2)}$ in $D_{(2)}$ with $\xi\left(t_{(2)}\right)>1$, so that by (14) we have that $\xi_{1}\left(t_{(2)}\right)>9 / 10$. This contradiction with (16) shows that no such component $D_{(2)}$ can exist, and completes the proof of (ii)".

Next, suppose that in fact $D^{\prime \prime}$ were branched. Then, given any point $d^{\prime \prime}$ in $D^{\prime \prime}\left(\subset D_{1}\right)$ there must exist two disjoint (apart from the point $\left.d^{\prime \prime}\right)$ paths $\Gamma_{1}, \Gamma_{2}$ from $d^{\prime \prime}$ to $\infty$ both of which lie in $D^{\prime \prime}$ and such that $\Gamma_{1}, \Gamma_{2}$ are not homotopic in $D^{\prime \prime}$. Let $D_{(3)}$ be that component of $D_{1}-\left(\Gamma_{1} \cup \Gamma_{2}\right)$ such that $\partial D_{1} \cap \partial D_{(3)}=\emptyset$; since $D^{\prime \prime}$ is assumed branched, it follows that $D_{(3)} \notin D^{\prime \prime}$.

Hence, using the local behaviour of harmonic functions we see that there must exist some point $t_{(3)}$ in $D_{(3)}$ with $\xi\left(t_{(3)}\right)<0$. Let $D_{(4)}$ be the component of $\{t: \xi(t)<0\}$ that contains $t_{(3)}$; since $D_{(4)}$ is connected, it follows that $\bar{D}_{(4)} \subset D_{(3)}$. Because $\xi(t)=0$ on $\partial D_{(4)}$ and $\xi(t)<0$ in $D_{(4)}$, we can deduce, using arguments similar to those in Theorem 1(a), (b), that $D_{(4)}$ must be unbounded and $\xi(t)$ must be unbounded below in $D_{(4)}$; in particular, there must exist some point $t_{(4)}$ in $D_{(4)}$ where $\xi\left(t_{(4)}\right)<-2$. But since $t_{(4)}$ lies in $D_{1}$ ve must have $\xi_{1}\left(t_{(4)}\right)>-1$, so that $\left|\xi\left(t_{(4)}\right)-\xi_{1}\left(t_{(4)}\right)\right|>1$ - which is impossible in view of (15). This contradiction completes the proof of (iii)".

Now we define the family of domains $\{D(c)\}$ by the following:

$$
D(c)=\left\{t: t \in D_{1}, \xi(t)>c\right\} \quad(c \geqq-9 / 10) .
$$

In the same way as done above for $D(0)=D^{\prime \prime}$, we can show that each set $D(c)$ is 
non-empty and unbounded, has its closure a subset of $D_{1}$, has only one component, and that component is unbranched.

In view of (15) we have that

where

$$
D_{1}(c+1 / 10) \subset D(c) \subset D_{1}(c-1 / 10) \quad(c \geqq-9 / 10),
$$

$$
D_{1}(c)=\left\{t: t>D_{1}, \xi_{1}(t)>c\right\} \quad(c \geqq-1) .
$$

The domain $D_{1}(c)$ contains the segment $\left[f_{1}(c+1 / 10),+\infty\right)$ of the real axis, and hence so does $D(c)$. For each $r>f_{1}(c+1 / 10)$ (and we must have $f_{1}(c+1 / 10)>0$ since $\left.f_{1}(c+1 / 10) \in \boldsymbol{R} \cap D_{1}\right)$, denote by $r \theta_{r}(c)$ the length of the arc of $\{t:|t|=r\} \cap D(c)$ that meets $\left[f_{1}(c+1 / 10),+\infty\right)$. Then the same argument that was used earlier for points of $\partial D_{1}^{\prime}$ to show that $\varepsilon(r) \rightarrow 0$ can be repeated for the points $r \exp \left((1 / 2) \theta_{r}(c)\right)$ on $\partial D(c)$ to show that $\theta_{r}(c) \rightarrow 2 \pi$ as $r \rightarrow \infty$. In particular, for the domain $D^{\prime \prime}=D(0)$ we have that $\theta_{r}(0) \rightarrow 2 \pi$ as $r \rightarrow \infty$.

3.3. The growth rate. We now examine the growth rate as $r \rightarrow \infty$ of

$$
M(r)=\max \left\{\xi(t): t \in D^{\prime \prime},|t|=r\right\} .
$$

In view of (15) we have that

$$
M(r)=M_{1}(r)+O(1),
$$

where $M_{1}(r)=\max \left\{\xi_{1}(t): t \in D^{\prime \prime},|t|=r\right\}$.

To begin with, however, we look at the growth rate of $\tilde{M}_{1}(r)=\max \left\{\xi_{1}(t)\right.$ : $\left.t \in D_{1},|t|=r\right\}$. Our aim is to show that $M(r), M_{1}(r)$ and $\tilde{M}_{1}(r)$ all $\sim(1 / L) r^{1 / 2}$ (as $r \rightarrow \infty$ ), where $L$ was given in (11).

Consider the point $t=r e^{i \varphi}$ (where $|\varphi|<\pi-\Theta(r)$ ) on $\{t:|t|=r\} \cap D_{1}$. As in Section 3.1 this point corresponds to a point, $x_{t}+i y_{t}$ say, in $D_{2}$ where

$$
x_{t}=\frac{1}{2} \log r \quad \text { and } \quad y_{t}=\frac{1}{2} \varphi .
$$

This latter point corresponds to a point, $u_{t}+i v_{t}$ say, in $D_{3}$ where (again using the methods of Section 3.1) we have that

$$
\left\{\begin{array}{l}
u_{t}=\lambda+o(1)+\pi \int_{0}^{x_{t}} \frac{d s}{\pi-(1 / 2) \Theta\left(e^{2 s}\right)} \\
v_{t}=o(1)+\pi \frac{y_{t}}{\pi-(1 / 2)} \overline{\Theta\left(e^{2 x_{t}}\right)}
\end{array}\right.
$$

where $\lambda$ is real and $o(1)$ is a term tending to zero as $x_{t}$ (and so $r$ ) tends to $+\infty$, uniformly in $y_{t}$ (and so in $\varphi$ ). The corresponding point in $D_{4}$ is $\xi_{t}+i \eta_{t}$, where

$$
\xi_{t}=e^{u_{t}} \cos v_{t}-1 \text { and } \eta_{t}=e^{u_{t}} \sin v_{t} .
$$

Then

$$
\tilde{M}_{1}(r) \max \left\{\xi_{t}: r \text { fixed, }|\varphi|<\pi-\Theta(r)\right\} .
$$


By exponentiating the first equation in (19) and following the same method as in Section 3.1, we see that for $t \in\{t:|t|=r\} \cap D_{1}$ we have

$$
e^{u_{t}}=r^{1 / 2} \exp \{\lambda+o(1)\} \exp \left\{\int_{1}^{r} \frac{(1 / 2 \pi) \Theta(\varrho)}{2 \varrho[1-(1 / 2 \pi) \Theta(\varrho)]} d \varrho\right\},
$$

where the $o$ (1) term in (22) is the same as that in (19), and so tends to zero as $r \rightarrow \infty$, uniformly in $\varphi$. Hence as $r \rightarrow \infty$ we have that $e^{u_{t}} \sim(1 / L) r^{1 / 2}$, uniformly in $\varphi$.

Now $\varphi$ can take all values between $\pm[\pi-\Theta(r)]$, and so $y_{t}$ can take all values between $\pm[\pi-\Theta(r)]$. Hence, as $r \rightarrow \infty$, (19) shows that $v_{t}$ can take all values between $\pm v_{r}$, say, where $0<v_{r}<\pi / 2$ and $v_{r} \rightarrow \pi / 2$ as $r \rightarrow \infty$.

Combining this observation with (20), (21) and (22) (with its uniformity in $\varphi$ ), we deduce that

$$
\left\{\begin{array}{c}
\tilde{M}_{1}(r) \sim r^{1 / 2} e^{\lambda+\mu /(4 \pi)}=\frac{1}{L} r^{1 / 2} \\
\sim f_{1}(r)=\xi_{1}(r) .
\end{array}\right.
$$

Since $r \in D^{\prime \prime}$ and $D^{\prime \prime} \subset D_{1}$, it follows that

$$
M_{1}(r) \sim \tilde{M}_{1}(r) \sim \xi_{1}(r) ;
$$

and finally, since $f_{1}(r) \rightarrow \infty$ as $r \rightarrow \infty,(17),(23)$ and (24) together give that

$$
M(r) \sim \xi_{1}(r) \sim \frac{1}{L} r^{1 / 2} .
$$

This completes the proof of Theorem 2.

Remark. In fact (25) is more precise than the result

$$
\lim _{r \rightarrow \infty} \log M(r) / \log r=\frac{1}{2}
$$

which we set out to prove.

\section{Completion of the proof of Theorem 2}

The details of the arguments in the construction of suitable functions in parts (b) - (e) of Theorem 2 are broadly similar to those in part (a), except that there is an additional mapping of the half-plane $D_{4}$ onto sectors of suitable angles.

Part (b). Choose an $n \geqq 2$ and any positive numbers $\varrho_{1}, \ldots, \varrho_{n}$ with $\sum_{k=1}^{n} 1 / \varrho_{k}=2$. Then using techniques similar to those in part (a) we can construct an entire function $f(t)=\xi(t)+i \eta(t)$ with the following properties:

(i) $\xi(t)>0$ in unbounded unbranched regions

$$
D_{1}, \ldots, D_{m} \text { and } \xi(t)=0 \text { on } \partial D_{1}, \ldots, \partial D_{m} ;
$$

(ii) $\xi(t)<0$ in unbounded unbranched regions

$$
D_{m+1}, \ldots, D_{n} \text { and } \xi(t)=0 \text { on } \partial D_{m+1}, \ldots, \partial D_{n} ;
$$


(iii) the order of $\xi(t)$ in the tract $D_{k}$ is $\varrho_{k}, 1 \leqq k \leqq n$; where category (i) or category (ii) can be empty, as desired, and $m$ can be arbitrarily chosen.

Firstly, we choose a sequence $\left\{\theta_{k}\right\}_{k=1}^{n}$ of real numbers and consider the closures of the domains

$$
S_{k}=\left\{t:\left|\arg \left(t-e^{i \theta_{k}}\right)\right|<\pi /\left(2 \varrho_{k}\right)\right\} \quad(1 \leqq k \leqq n) .
$$

Then for $1 \leqq k \leqq m$ define the function $f_{1}(t)$ in $\bar{S}_{k}$ to be any function mapping $\bar{S}_{k}$ one-one onto $\{\xi: \operatorname{Re} \xi \geqq-1\}$ with $f_{1}\left(e^{i \theta_{k}}\right)=-1$, and for $m+1 \leqq k \leqq n$ define $f_{1}$ in $\bar{S}_{k}$ to be any function mapping $\bar{S}_{k}$ one-one onto $\{\xi: \operatorname{Re} \xi \leqq+1\}$ with $f_{1}\left(e^{i \theta_{k}}\right)=1$. Next we approximate, using Arakelian's theorem, to $f_{1}$ in $\bigcup_{k=1}^{n} \bar{S}_{k}$ by an entire function $f(t)=\xi(t)+i \eta(t)$ in the same way as in part (a); and, as before, there exist tracts in the plane in which $\xi(t)$ has the properties (i), (ii) and (iii).

Part (c). Choose any infinite sequence of positive numbers $\left\{\varrho_{k}\right\}_{k=1}^{n}$ with $\sum_{k=1}^{n} 1 / \varrho_{k}=2$. The construction is similar to that in part (b), except that we have to ensure that no finite point has a neighbourhood that meets more than one $S_{k}$. Therefore we choose sectors $S_{k}$ of opening $\pi / \varrho_{k}$ with radial midlines and with vertices at the points $r_{k} e^{i \theta_{k}}$ where $r_{k}=1+k, \theta_{1}=0$ and $\theta_{k+1}=\theta_{k}+\pi /\left(2 \varrho_{k}\right)+\pi /\left(2 \varrho_{k+1}\right)(k \geqq 1)$. The same mapping-and-approximation method then works successfully.

Part (d). Notice that the result of part (b) is that of part (d) when $\sigma=2$. In view of Theorem 1(c) we know that the order of the final harmonic function $\xi(t)$ must be inf nite in all tracts except those associated with the sectors $S_{k}$ in our construction; when $\sigma<2$ we have to be careful to ensure this is still so.

Firstly for given $\varrho_{k}$ and $\sigma$ we define the sectors

$$
S_{k}=\left\{t:\left|\arg \left(t-e^{i \theta_{k}}\right)\right|<\pi /\left(2 \varrho_{k}\right)\right\} \quad(1 \leqq k \leqq n),
$$

where $\theta_{1}=\pi /\left(2 \varrho_{1}\right)$ and $\theta_{k+1}=\theta_{k}+\pi /\left(2 \varrho_{k+1}\right)(1 \leqq k<n)$. All the sectors $S_{k}$ lie in the sector

$$
S_{(\sigma)}=\{t: 0<\arg t<\sigma \pi\},
$$

and are disjoint; in fact the set

$$
\left(S_{(\sigma)}-\bigcup_{k=1}^{n} S_{k}\right) \cap\{t:|t|>1\}
$$

consists of $(n+1)$ half-strips whose sides are parallel and whose 'ends' are arcs of $\{t:|t|=1\}$.

Next we define the open sector

$$
D_{(\sigma)}=C-\overline{S_{(\sigma)}}
$$

this has axis of symmetry

$$
A=\{t: \arg t=-(1-\sigma / 2) \pi\},
$$

and is convex in the direction of $A$. Now define point sets ('chevrons') $\left\{T_{m}\right\}_{m=0}^{\infty}$ and the 
set $E$ as follows:

and

$$
\begin{aligned}
& T_{0}=\partial D_{(\sigma)}, \\
& T_{m}=\left\{t: t+m e^{-(1-\sigma / 2) \pi} \in T_{0}\right\} \quad(m \in N),
\end{aligned}
$$

$$
E=\left(\bigcup_{k=1}^{n} \bar{S}_{k}\right) \cup\left(\bigcup_{m=0}^{\infty} T_{m}\right) ;
$$

$E$ is closed in $C$, and its complement is connected and 'connected at $\infty$ ' (in the sense of Arakelian).

Now we define the function $\xi_{1}(t)$ on $E$ as follows:

$$
\xi_{1}(t)= \begin{cases}(-1)^{m+1} & \left(t \in T_{m}, m \geqq 0\right) \\ \xi_{j}(t) & \left(t \in \bar{S}_{j}, \quad j \geqq 1\right),\end{cases}
$$

where $\xi_{j}(t)$ is the real part of any analytic function that maps $\bar{S}_{j}$ one-one onto the half-plane $\{\xi: \operatorname{Re} \xi \geqq-1 / 2\}$. By Arakelian's theorem there exists a function $\xi(t)$ harmonic in the whole plane such that

$$
\left|\xi(t)-\xi_{1}(t)\right|<\frac{1}{10} \quad(t \in E)
$$

Then the components of $\{t: \xi(t) \neq 0\}$ are all of one of the following types:

(i) subsets of $S_{k}(1 \leqq k \leqq n)$;

(ii) contain a $T_{m}$ but disjoint from $T_{m-1}$ and $T_{m+1}(m>1)$;

(iii) lie between some $T_{m}$ and $T_{m+1}$;

(iv) lie in $S_{(\sigma)}-\left(\bigcup_{k=1}^{n} S_{k}\right)$.

Arguments similar to those in Section 3 show that there are precisely $n$ tracts of type (i), and the order of $\xi(t)$ in these is $\varrho_{1}, \ldots, \varrho_{n}$; and a straightforward application of our Lemma ensures that $\xi(t)$ has infinite order in all the other tracts. This completes part (d).

Part $(e)$. Here we are given $\sigma \in(0,2)$ and $\varrho_{k}>0$ with $\sum_{k=1}^{\infty} 1 / \varrho_{k}=\sigma$.

For each $k \geqq 1$ we construct a sector $S_{k}$ as follows:

$$
S_{k}=\left\{t:\left|\arg \left(t-r_{k} e^{i \theta_{k}}\right)\right|<\pi / \varrho_{k}\right\},
$$

where $r_{k}=k, \theta_{1}=\pi / 2 \varrho_{1}$ and

$$
\theta_{k+1}=\theta_{k}+\frac{\pi}{2 \varrho_{k}}+\frac{\pi}{2 \varrho_{k+1}} \quad(k \geqq 1) .
$$

All the $S_{k}$ lie in the sector

$$
S_{(\sigma)}=\{t: 0<\arg t<\sigma \pi\}
$$

and subtend at 0 an angle of $\{t:|t|=r\}$ which tends to 0 as $r \rightarrow \infty$. The $S_{k}$ are disjoint from each other and from the complement of $S_{(\sigma)}$.

We can then 'fill' the complement of $S_{(\sigma)}$ with 'chevrons' $\left\{T_{m}\right\}_{m=0}^{\infty}$, as described in part (d), and the rest of the argument follows as above. 
Remark. In the case where $\sigma=0$ in Theorem 2, we note that the function

$$
\xi(t)=\operatorname{Re}\left(e^{t}\right)
$$

has infinite order in each tract $\{t: \xi(t) \neq 0\}$.

\section{Proof of Theorem 3}

This follows exactly as in [1; pp. 366-367], once we have proved

Lemma 2. With the hypotheses on the domain $D$ of Theorem 3, there exists a function $v(z)$ harmonic in $D$ such that

$$
v(z) \geqq \varphi(|z|) \quad(z \in D) .
$$

Proof of Lemma 2. We use the notation and method of [1; Lemma 1, p. 364], with $F$ there replaced by $\partial D$. The argument starts out in the same way; as before the key step is to show that the $v(z)$ in equation (2.3) of [1] is not identically $+\infty$, by finding some upper bound on $v\left(z_{0}\right)$ at some point $z_{0}$ in $D$.

As in [1], we have that

$$
\begin{aligned}
v_{R_{n}}(z) & \leqq \sum_{v=K}^{n-1} \varphi\left(R_{v+1}\right) \omega_{v, n}(z)+\varphi\left(R_{K}\right) \\
& \leqq \sum_{v=K}^{n-1} \varphi\left(R_{v+1}\right) \omega_{v}(z)+\varphi\left(R_{K}\right) ;
\end{aligned}
$$

but now we estimate $\omega_{v}$ not by the Milloux-Schmidt inequality but by

Lemma 1. Thence, with $\varkappa=1 / 2$, we deduce that

$$
\omega_{v}\left(z_{0}\right) \leqq \frac{9}{(1 / 2)^{1 / 2}} \exp \left[-\pi \int_{2\left|z_{0}\right|}^{R_{v} / 2} \frac{d \sigma}{\sigma \theta(\sigma)}\right],
$$

so long as $\left|z_{0}\right|<R_{v} / 4$; hence we choose $K$ so that $R_{K}>4\left|z_{0}\right|+r_{0}+2 r_{1}$. obtain

Using hypothesis $(c)$ of Theorem 3 , we can bound the right-hand-side of (27) to

$$
\omega_{v}\left(z_{0}\right) \leqq A\left(z_{0}, r_{1}\right) R_{v}^{-1 / \alpha}
$$

where, $A\left(z_{0} r_{1}\right)$ is some constant independent of $v$; it follows from (26) and (28) that

Now,

$$
v_{R_{n}}\left(z_{0}\right) \leqq A\left(z_{0}, r_{1}\right) \sum_{v=K}^{n-1} \varphi\left(R_{v+1}\right) R_{v}^{-1 / \alpha}+O(1) .
$$

$$
\begin{aligned}
\int_{R_{v+1}}^{R_{v+2}} \frac{\varphi(t)}{t^{1+1 / \alpha}} d t & \geqq \varphi\left(R_{v+1}\right) \int_{R_{v+1}}^{R_{v+2}} \frac{d t}{t^{1+1 / \alpha}} \\
& \geqq \varphi\left(R_{v+1}\right) A_{\alpha}\left\{R_{v+1}^{-1 / \alpha}-R_{v+2}^{-1 / \alpha}\right\} \\
& \geqq \varphi\left(R_{v+1}\right) A_{\alpha} R_{v}^{-1 / \alpha},
\end{aligned}
$$

where $A_{\alpha}$ is a constant depending on $\alpha$ (and not necessarily the same on each occur- 
rence), and where we have used the fact that $R_{v}=2^{v}$. It follows from (29) and (30) that

$$
\begin{aligned}
v_{R_{n}}\left(z_{0}\right) & \leqq A_{\alpha} A\left(z_{0}, r_{1}\right) \sum_{v=K}^{n-1} \int_{R_{v+1}}^{R_{v+2}} \frac{\varphi(t)}{t^{1+1 / \alpha}} d t+O(1) \\
& \leqq A_{\alpha} A\left(z_{0}, r_{1}\right) \int_{R_{K+1}}^{\infty} \frac{\varphi(t)}{t^{1+1 / \alpha}} d t+O(1)
\end{aligned}
$$

as the upper bound in (31) is independent of $n$, the proof of Lemma 2 (and thus of Theorem 3) is complete.

\section{An example related to Theorem 3}

In the opposite direction to Theorem 3 we now prove

Theorem 4. Let $\beta \in(0,2)$, and let $\psi(t)$ be a positive increasing function on $[0, \infty)$ with

$$
\lim _{t \rightarrow+\infty} \psi(t)=+\infty, \int_{1}^{\infty} \frac{\psi(t)}{t^{1+1 / \beta}} d t=+\infty .
$$

Then there exists a function $u(z)$ harmonic in $C$ with the following properties:

(a) In every tract $T$ in the family $\{z: u(z) \neq 0\}$, we have

$$
\limsup _{r \rightarrow \infty} \frac{1}{\log r} \int_{r_{1}}^{r} \frac{d \sigma}{\sigma \theta(\sigma)} \geqq \frac{1}{\beta \pi}
$$

for some value of $r_{1}$, where $\sigma \theta(\sigma)$ denotes the length of the set $\operatorname{T\cap }\{z:|z|=\sigma\}$.

(b) On every path $\Gamma$ going to $\infty$ on which $|u(z)| \rightarrow \infty$, we have

$$
\liminf _{z \rightarrow \infty, z \in \Gamma} \frac{|u(z)|}{\psi(|z|)}<+\infty .
$$

Remark. Theorem 2 of [1] is essentially the case $\beta=2$ of this result.

Proof of Theorem 4. This follows the same pattern as the proof of Theorem 2 of [1; see pp. 368-384]. We indicate here only the necessary changes in the argument, and we use the same notation as in [1].

The general idea is that Theorem 4 on page 368 of [1] still holds for our function $\psi$, but the extension domain $D$ must be different so that conclusion (a) will hold. Then in the proof of the Corollary on page 368 of [1] we do not use Mergelyan's theorem and arrange to have a harmonic polynomial that approximates only on the extension domain; instead we approximate on the extension domain and to a constant \pm 1 at other specified parts of the plane, using Arakelian's theorem. Later we use Mergelyan's theorem on a very much larger domain so that we can approximate Arakelian's entire function by a harmonic polynomial, ready for the iterative application of Theorem 4 of [1]. 
To begin with, we use the basic approximation results of Sections 3.1 and 3.2 of [1], as well as the construction of a $D_{1}$ in Section 4 of [1].

In Section 4.1 of [1] we make the following different choices for Q's:

$$
Q_{2 \mu}=R_{\mu} e^{-i \beta \pi / 4}, \quad Q_{2 \mu+1}=R_{\mu} e^{i \beta \pi / 4} .
$$

Then the choices of $S$ ' $s$ and $T$ 's is such that the sectorial region in (4.3) of [1] is replaced by

$$
S_{\mu}=\left\{z: R_{\mu}<|z|<R_{\mu}^{\prime}, \quad|\arg z|<\beta \pi / 2-\eta_{\mu+1}\right\} ;
$$

otherwise the discussion of the extension domain and the harmonic functions on it is much the same.

The argument in Section 5 of [1] is unaltered, down to (5.3). On this occasion (5.3) is still valid, where this time we apply Harnack's inequality [4; p. 31] to the function $V_{\mu}$, the compact set $\left\{0,9 R_{\mu}\right\}$, and the domain $R=\left(D_{2}-\Delta_{1}\right) \cup \Delta_{2}$, where

and

$$
\Delta_{1}=\left(D^{(\mu+1)}-D^{(\mu)}\right) \cap\left\{z:|z| \leqq R_{\mu}\right\}
$$

$$
\Delta_{2}=\left\{z: R_{\mu}<|z|<100 R_{\mu},|\arg z|<\beta \pi / 4\right\} .
$$

Then in an argument analogous to that on page 384 of [1], we choose an $R_{\mu}^{\prime}>$ $100 R_{\mu}$ such that

$$
\frac{\left(9 R_{\mu}\right)^{1 /(2 \beta)}}{2(\beta \pi)} \int_{9 R_{\mu}}^{R_{\mu}^{\prime} / 9} \frac{\psi(r)}{r^{1+1 / \beta}} d r>M_{2} C_{\mu} .
$$

Now we choose a $\beta_{1}$ with $3 \beta / 4<\beta_{1}<\beta$ such that

$$
\frac{\left(9 R_{\mu}\right)^{1 /\left(2 \beta_{1}\right)}}{2\left(\beta_{1} \pi\right)} \int_{9 R_{\mu}}^{R_{\mu}^{\prime} / 9} \frac{\psi(r)}{r^{1+1 / \beta_{1}}} d r>M_{2} C_{\mu},
$$

and define $\eta_{\mu+1}$ by the formula

$$
\beta_{1} \pi=\beta \pi-2 \eta_{\mu+1} .
$$

Applying Lemma 6 of [1] to (5.3) then shows that

so that again $\alpha_{\mu}<1$.

$$
V_{\mu}\left(9 R_{\mu}\right) \geqq \frac{\left(9 R_{\mu}\right)^{1 /\left(2 \beta_{1}\right)}}{2\left(\beta_{1} \pi\right)} \int_{9 R_{\mu}}^{R_{\mu}^{\prime} / 9} 2 \alpha_{\mu} \frac{\psi(r)}{r^{1+1 / \beta_{1}}} d r>\alpha_{\mu} M_{2} C_{\mu} ;
$$

The important points to note in the above argument are that our sectorial region replacing that in (4.3) of [1] has an angular opening $\beta \pi-2 \eta_{\mu+1}<\beta \pi$, and that the argument only requires that $R_{\mu}^{\prime}$ be larger than some minimum size - for any additional condition that we wish to put on the minimum size of $R_{\mu}^{\prime}$ all the above argument will still apply. We also need to arrange that

$$
\log R_{\mu}<\left(\log R_{\mu}^{\prime}\right)^{1 / 2} .
$$

With $S_{\mu}$ defined by (32) and $T_{\mu}$ defined to be

$$
T_{\mu}=\left\{z: R_{\mu}<|z|<R_{\mu}^{\prime},|\arg (-z)|<(1-\beta / 2) \pi\right\},
$$

both $S_{\mu}$ and $T_{\mu}$ lie in the annulus $\left\{z: R_{\mu}<|z|<R_{\mu}^{\prime}\right\}$, and they have disjoint closures. 
Now we have to prove the analogue of the Corollary on page 368 of [1], with the additional requirement (a) of our Theorem 4 on the components of $\{z: v(z) \neq 0\}$; for then the proof of the present Theorem 4 follows immediately.

Suppose that at some stage of the construction we have a harmonic polynomial $v_{n}(z)$, and that we choose some suitably large $R$ so that after applying Theorem 4 of [1] all our extensions lie in $\{z:|z|<R / 2\}$.

We then construct an extension domain $D$ associated with the disc $\{z:|z|<R\}$, as described at the beginning of this proof. For $1 \leqq \mu \leqq N$, let $T_{\mu}^{\prime}$ denote $T_{\mu}$ together with a Jordan curve $J_{\mu}$ joining $-R_{\mu}$ to $\infty$ chosen in such a way that all the $J_{\mu}$ are disjoint and meet no other $T_{v}(v \neq \mu)$ or $\bar{D}$. Then we define $V_{n}$ as follows:

$$
V_{n}(z)=\left\{\begin{array}{cc}
v_{n}(z) & (z \in \bar{D}) \\
-1 & \left(z \in T^{\prime}=\bigcup_{\mu=1}^{N} T_{\mu}^{\prime}\right) .
\end{array}\right.
$$

We can now apply Arakelian's approximation theorem to the function $V_{n}$ defined on the set $\bar{D} \cup T^{\prime} . V_{n}$ is harmonic on the interior of this set and is continuous on its closure; the curves $J_{\mu}$ may be chosen such that $C-\left(\bar{D} \cup T^{\prime}\right)$ is connected at $\infty$, and from our construction $C-\left(\bar{D} \cup T^{\prime}\right)$ is connected.

Hence by Arakelian's theorem there exists a function $W_{n}(z)$ harmonic in $C$ such that

$$
\left|W_{n}(z)-V_{n}(z)\right|<\frac{1}{4} \varepsilon_{n} \quad\left(z \in \bar{D} \cup T^{\prime}\right) .
$$

Next, by Mergelyan's theorem there exists a harmonic polynomial, $v_{n+1}(z)$ say, such that

where $M=2 \sup \{|z|: z \in \bar{D}\}$.

$$
\left|W_{n}(z)-v_{n+1}(z)\right|<\frac{1}{4} \varepsilon_{n} \quad(|z| \leqq M) .
$$

It follows that for $z \in \bar{D}$,

$$
\begin{aligned}
\left|v_{n+1}(z)-v_{n}(z)\right| & =\left|V_{n}(z)-v_{n+1}(z)\right| \\
& \leqq\left|V_{n}(z)-W_{n}(z)\right|+\left|W_{n}(z)-v_{n+1}(z)\right| \\
& <\frac{1}{2} \varepsilon_{n} .
\end{aligned}
$$

Then, as on page 369 of [1] it follows that a sequence $\left\{v_{n}(z)\right\}$ defined in this way, where we apply Theorem 4 of [1] successively to $(-1)^{n} v_{n}(z)$, converges to a function $u(z)$ harmonic in $C$ that satisfies conclusion (b).

Now let $T$ be any component of the set $\{z: u(z) \neq 0\}$. Then if we take $\varepsilon<1$ on page 369 of [1], it follows from our construction that there exist sequences $\left\{R_{\mu}\right\}_{1}^{\infty}$ and $\left\{R_{\mu}^{\prime}\right\}_{1}^{\infty}$ going to $\infty$ such that $T$ does not meet any of the sets $T_{\mu}$ defined by (34).

From our construction we have that $\theta(\sigma) \leqq \beta \pi$ for $R_{\mu}<\sigma<R_{\mu}^{\prime}$, so that

$$
\int_{R_{\mu}}^{R_{\mu}^{\prime}} \frac{d \sigma}{\sigma \theta(\sigma)} \geqq \frac{1}{\beta \pi}\left(\log R_{\mu}^{\prime}-\log R_{\mu}\right) ;
$$


consequently we may use our hypothesis (33) to deduce that

$$
\begin{aligned}
\frac{1}{\log R_{\mu}^{\prime}} \int_{R_{\mu}}^{R_{\mu}^{\prime}} \frac{d \sigma}{\sigma \theta(\sigma)} & \geqq \frac{1}{\beta \pi}-\frac{1}{\beta \pi} \cdot \frac{\log R_{\mu}}{\log R_{\mu}^{\prime}} \\
& >\frac{1}{\beta \pi}-\frac{1}{\pi \beta} \cdot \frac{1}{\left(\log R_{\mu}^{\prime}\right)^{1 / 2}} \\
& \rightarrow \frac{1}{\beta \pi} \quad(\text { as } \mu \rightarrow \infty) .
\end{aligned}
$$

Hence conclusion (a) of our Thesrem is satisfied, and our proof is complete.

\section{References}

[1] Barth, K. F., D. A. Brannan, and W. K. HaYman: The growth of plane harmonic functions along an asymptotic path. - Proc. London Math. Soc. (3) 37, 1978, 363-384.

[2] Fuchs, W. H. J.: Théorie de l'approximation des fonctions d'une variable complexe. - Séminaire de Mathématiques Supérieures, No. 26, (Été, 1967). Les Presses de l'Université de Montréal, Montréal, Canada, 1968.

[3] HeINs, M. H.: On a notion of convexity connected with a method of Carleman. - J. Analyse Math. 7, 1959, 53-77.

[4] Helms, L. L.: Introduction to potential theory. - Wiley-Interscience, New York-LondonSydney, 1969.

[5] KJELlberg, B.: On the growth of minimal positive harmonic functions in a plane region. - Ark. Mat. 1, 1950, 347-351.

[6] LindelöF, E., and E. PhragmÉn: Sur une extension d'un principe classique de l'analyse et sur quelques propriétés des fonctions monogènes dans le voisinage d'une point singulier. Acta Math. 31, 1908, 381-406.

[7] TALPUR, M. N. M.: A subharmonic analogue of Iversen's theorem. - Proc. London Math. Soc. (3) $31,1975,129-148$.

[8] Titchmarsh, E. C.: The theory of functions, second edition. - Oxford University Press, Oxford, 1939.

[9] Tsuj, M.: Potential theory in modern function theory. - Maruzen, Co., Ltd., Tokyo, 1959.

[10] Tsuju, M.: A theorem on the majoration of harmonic measure and its applications. - Tôhoku Math. J. 3, 1951, 13-23.

[11] Warschawski, S. E.: On conformal mapping of infinite strips. - Trans. Amer. Math. Soc. 51, $1942,280-335$.

Syracuse University

Department of Mathematics

Syracuse, New York 13210

USA

Received 28 February 1985

\author{
The Open University \\ Faculty of Mathematics \\ Walton Hall \\ Milton Keynes MK7 6AA \\ England
}

